\title{
Treatment of symptomatic diabetic peripheral neuropathy with the anti-oxidant $\alpha$-lipoic acid
}

\section{A 3-week multicentre randomized controlled trial (ALADIN Study)}

\author{
D. Ziegler ${ }^{1}$, M. Hanefeld ${ }^{2}$, K. J. Ruhnau ${ }^{3}$, H.P.Meißner ${ }^{3}$, M.Lobisch ${ }^{4}$, K. Schütte ${ }^{4}$, F. A. Gries ${ }^{1}$, \\ The ALADIN Study Group* \\ ${ }^{1}$ Diabetes-Forschungsinstitut an der Heinrich-Heine-Universität, Düsseldorf, Germany \\ ${ }^{2}$ Abteilung Klinische Stoffwechselforschung, Universitätsklinikum "Carl Gustav Carus" der Technischen Universität, Dresden, \\ Germany \\ ${ }^{3}$ Diabetologische Schwerpunktpraxen, Berlin, Germany \\ ${ }^{4}$ Medizinische Forschung, ASTA Medica AG, Frankfurt am Main, Germany
}

Summary Anti-oxidant treatment has been shown to prevent nerve dysfunction in experimental diabetes mellitus, thus providing a rationale of potential therapeutic value for diabetic patients. The effects of the anti-oxidant $\alpha$-lipoic acid (thioctic acid) were studied in a 3-week multicentre, randomized, doubleblind placebo-controlled trial (Alpha-Lipoic Acid in Diabetic Neuropathy; ALADIN) in 328 non-insulindependent diabetic patients with symptomatic peripheral neuropathy who were randomly assigned to treatment with intravenous infusion of $\alpha$-lipoic acid using three doses $(1200,600$, or $100 \mathrm{mg}$ ALA) or placebo (PLAC). Neuropathic symptoms (pain, burning, paraesthesiae, and numbness) were scored at baseline and at each visit (days 2-5, 8-12, and 15-19) prior to infusion. In addition, the Hamburg Pain Adjective List, a multidimensional specific pain questionnaire, and the Neuropathy Symptom and Disability Scores were assessed at baseline and day 19. According to the protocol $260(65 / 63 / 66 / 66)$ patients completed the study. The total symptom score in the feet decreased from baseline to day 19 by $-4.5 \pm 3.7$ $(-58.6 \%)$ points (mean \pm SD) in ALA 1200,
$-5.0 \pm 4.1(-63.5 \%)$ points in ALA $600,-3.3 \pm 2.8$ $(-43.2 \%)$ points in ALA 100, and $-2.6 \pm 3.2$ $(-38.4 \%)$ points in PLAC (ALA 1200 vs PLAC: $p=0.003$; ALA 600 vs PLAC: $p<0.001)$. The response rates after 19 days, defined as an improvement in the total symptom score of at least $30 \%$, were $70.8 \%$ in ALA $1200,82.5 \%$ in ALA 600, $65.2 \%$ in ALA 100, and 57.6\% in PLAC (ALA 600 vs PLAC; $p=0.002$ ). The total scale of the Pain Adjective List was significantly reduced in ALA 1200 and ALA 600 as compared with PLAC after 19 days (both $p<0.01$ ). The rates of adverse events were $32.6 \%$ in ALA $1200,18.2 \%$ in ALA $600,13.6 \%$ in ALA 100, and $20.7 \%$ in PLAC. These findings substantiate that intravenous treatment with $\alpha$-lipoic acid using a dose of $600 \mathrm{mg} /$ day over 3 weeks is superior to placebo in reducing symptoms of diabetic peripheral neuropathy, without causing significant adverse reactions. [Diabetologia (1995) 38: 1425-1433]

Key words Diabetic neuropathy, symptom scores, treatment, anti-oxidants, $\alpha$-lipoic acid.
Received: 31 March 1995 and in revised form: 5 June 1995

* A list of the participants in the ALADIN Study appears in the acknowledgements

Corresponding author: Dr. D. Ziegler, Diabetes-Forschungsinstitut an der Heinrich-Heine-Universität, Auf'm Hennekamp 65, D-40225 Düsseldorf, Germany

Abbreviations: ALA, $\alpha$-lipoic acid; HPAL, Hamburg Pain Adjective List; NCV, nerve conduction velocity; NDS, Neuropathy Disability Score; NSS, Neuropathy Symptom Score; TSS, total symptom score; VPT, vibration perception threshold; NIDDM, non-insulin-dependent diabetes mellitus; IDDM, insulin-dependent diabetes mellitus.
Near-normoglycaemia is now generally accepted as the primary goal in prevention of diabetic neuropathy $[1,2]$. However, in diabetic patients with advanced stages of peripheral neuropathy relatively long periods of near normal glycaemic control for several months or even years may be needed to retard the progression of nerve dysfunction [3, 4]. Hence, in symptomatic diabetic neuropathy additional pharmacological treatment of painful neuropathic symptoms is frequently required to maintain quality of life. Although treatment of pain with tricyclic antidepressants such as amitriptyline is effective $[5,6]$, it 
may be of limited value due to frequent adverse reactions [5] or increased risk of mortality from overdose [7]. Other symptomatic approaches including anticonvulsants [6], mexiletine [8], and topical capsaicin [9] have either not been unequivocally effective [6], have shown only partial effects [8], or caution has been urged as to potential neurotoxic side effects in view of longer-term treatment [10]. Furthermore, these medications are only designed to modulate symptoms without influencing the underlying neuropathy.

Potential forms of treatment that have emerged from the current concepts on the pathogenesis of diabetic neuropathy include the reduction of increased flux through the polyol pathway using aldose reductase inhibitors [11, 12], substitution of myo-inositol [13], inhibition of the formation of advanced glycation end products by aminoguanidine [14], correction of depleted neurotrophic factors by nerve growth factor substitution [15], elimination by vasodilators of endoneurial hypoperfusion resulting in hypoxia [16], correction of alterations in essential fatty acid metabolism by gamma-linolenic acid [17], and substitution of acetyl-L-carnitine [18]. Although some of these experimental therapeutic approaches have been investigated in human diabetic neuropathy, their efficacy has not yet been unequivocally established and in any case, these medications are not available in most countries.

There is accumulating evidence suggesting that free-radical-mediated oxidative stress is implicated in the pathogenesis of diabetic neuropathy by inducing neurovascular defects that result in endoneurial hypoxia and subsequent nerve dysfunction [1921]. Administration of physiological anti-oxidants including $\alpha$-lipoic acid, a potent lipophilic free radical scavenger [22], resulted in prevention of neurovascular abnormalities associated with experimental diabetic neuropathy [23-27], providing a basis of potential therapeutic value in diabetic patients. In fact, lipid-soluble anti-oxidants have recently been singled out as potential candidates for clinical studies [21].

In a randomized single-blind pilot study we have previously demonstrated an improvement in symptoms of diabetic neuropathy during a 3-week period of intravenous treatment with $600 \mathrm{mg}$ of $\alpha$-lipoic acid per day as compared with vitamin $B_{1}[28]$. This finding in conjunction with recent experimental evidence [27] formed the rationale for a large-scale, multicentre, double-blind placebo-controlled trial designed to assess the efficacy and safety of three different doses of $\alpha$-lipoic acid in non-insulin-dependent (NIDDM) diabetic out-patients with symptomatic peripheral neuropathy.

\section{Subjects and methods}

Study design. The Alpha-Lipoic Acid in Diabetic Neuropathy Study (ALADIN) was a randomized, double-blind placebocontrolled trial using three doses of the trometamol salt solution of $\alpha$-lipoic acid (Thioctacid T, ASTA Medica, Frankfurt am Main, Germany) in four parallel treatment groups of NIDDM patients with symptomatic peripheral neuropathy. Intravenous infusion of $1200 \mathrm{mg}$ (ALA 1200), $600 \mathrm{mg}$ (ALA 600), $100 \mathrm{mg}$ (ALA 100) $\alpha$-lipoic acid, or placebo in $250 \mathrm{ml} 0.9 \%$ isotonic saline solution over $30 \mathrm{~min}$ was administered once daily over two 5-day periods (Monday to Friday) and one 4-day period (Monday to Thursday) during 3 consecutive weeks. Ampules containing $10 \mathrm{ml}(250 \mathrm{mg})$ and $4 \mathrm{ml}$ $(100 \mathrm{mg}) \alpha$-lipoic acid or placebo were used. Each patient received six ampules containing $48 \mathrm{ml}$ in total (four ampules with $10 \mathrm{ml}$ each and two ampules with $4 \mathrm{ml}$ each): ALA 1200: $40 \mathrm{ml}(1000 \mathrm{mg})$ and $8 \mathrm{ml}(200 \mathrm{mg})$; ALA $600: 20 \mathrm{ml}(500 \mathrm{mg})$, $4 \mathrm{ml}(100 \mathrm{mg})$, and $24 \mathrm{ml}$ placebo; ALA $100: 4 \mathrm{ml}(100 \mathrm{mg})$ and $44 \mathrm{ml}$ placebo; placebo: $48 \mathrm{ml}$ placebo. Because of the yellow colour of the $\alpha$-lipoic acid containing solutions, riboflavin $(0.00375 \mathrm{mg} / \mathrm{ml})$ was added to the placebo to obtain a preparation of the same colour. Thus, the colour of the solutions containing the three doses of $\alpha$-lipoic acid and placebo was identical.

The study was designed as a confirmatory comparison of the group treated with $600 \mathrm{mg} \alpha$-lipoic acid with placebo after 19 days using a total symptom score as the primary endpoint.

Subjects. After approval by the ethical committee of the Landesärztekammer, Brandenburg, Germany, and written informed consent had been obtained, 328 patients were recruited from 38 out-patient centres in Germany. They were randomly assigned to receive one of four treatments: $\alpha$-lipoic acid $1200 \mathrm{mg}$ (ALA 1200), $600 \mathrm{mg}$ (ALA 600), $100 \mathrm{mg}$ (ALA 100), or placebo according to their entry sequence following a central computerized randomization list. All investigators and patients were "blinded" to the randomization of the study drug assignments.

Patients were eligible if they were aged 18 years and over and less than 70 years, had NIDDM treated with diet, oral anti-diabetic agents and/or insulin, and had evidence of symptomatic symmetrical distal neuropathy (reduced/absent ankle reflexes, reduced vibration, thermal, tactile, pin-prick, and/or position sensation) with at least moderate severity of one or more of the typical symptoms (burning, paraesthesiae, numbness) in the feet equivalent to 2 or more points in the total symptom score (outlined in Methods). Exclusion criteria were: 1) severe neuropathy including paresis, muscle atrophy, and very severe sensory deficits with reduced vibration or thermal sensation at five or six testing sites, respectively; 2) peripheral vascular disease (non-palpable foot pulses, intermittent claudication); 3) causes of neuropathy other than diabetes (e.g. chronic alcohol abuse, drug-induced neuropathy), truncal neuropathy, and significant neurological diseases (e.g. Parkinson's disease, epilepsy, multiple sclerosis); 4) use of medication likely to interfere with the interpretation of the results (e.g. antidepressants, anticonvulsants, opiates, mexiletine, capsaicin, neuroleptics, vitamin B compounds, gamma-linolenic acid, aldose reductase inhibitors, anti-oxidants; 5) participation in another study; 6) severe concomitant diseases (e.g. malignancies, hepatic or renal disease); 7) pregnancy, lactation, or childbearing age without safe contraception.

The baseline clinical characteristics of the four groups studied according to the protocol are shown in Table 1. As a sign of homogeneity of the groups formed by the randomization process no significant differences between them were noted for any of the variables listed. 
Table 1. Clinical characteristics of the patients at entry into the study

\begin{tabular}{|c|c|c|c|c|}
\hline & $\alpha$-Lipoic acid $1200 \mathrm{mg}$ & $\alpha$-Lipoic acid $600 \mathrm{mg}$ & $\alpha$-Lipoic acid $100 \mathrm{mg}$ & Placebo \\
\hline Number & 65 & 63 & 66 & 66 \\
\hline Sex (male/female) & $40 / 60$ & $37 / 63$ & $51 / 49$ & $35 / 65$ \\
\hline Body mass index $\left(\mathrm{kg} / \mathrm{m}^{2}\right)$ & $29.2 \pm 4.8$ & $27.7 \pm 4.9$ & $27.8 \pm 4.4$ & $29.7 \pm 4.9$ \\
\hline Systolic blood pressure $(\mathrm{mm} \mathrm{Hg})$ & $144 \pm 16$ & $143 \pm 16$ & $145 \pm 15$ & $144 \pm 14$ \\
\hline Duration of diabetes (years) & $11.0 \pm 7.3$ & $10.4 \pm 7.1$ & $11.7 \pm 7.0$ & $12.3 \pm 7.7$ \\
\hline Insulin treatment $\mathrm{t}^{\mathrm{a}}$ & 57 & 67 & 68 & 65 \\
\hline Blood glucose (mmol/l) & $11.1 \pm 4.1$ & $11.2 \pm 4.1$ & $11.4 \pm 4.3$ & $11.0 \pm 3.6$ \\
\hline $\mathrm{HbA}_{1}(\%)$ & $8.8 \pm 1.9$ & $9.2 \pm 2.5$ & $9.0 \pm 2.1$ & $9.4 \pm 2.6$ \\
\hline Retinopathy & 34 & 30 & 27 & 38 \\
\hline Neuropathy Symptom Score & $5.3 \pm 1.8$ & $5.6 \pm 2.0$ & $5.0 \pm 1.9$ & $5.3 \pm 1.7$ \\
\hline Neuropathy Disability Score & $6.1 \pm 2.7$ & $6.0 \pm 2.5$ & $6.2 \pm 2.6$ & $6.2 \pm 2.4$ \\
\hline Pain Adjective List (total scale) & $2.01 \pm 1.44$ & $2.14 \pm 1.51$ & $2.17 \pm 1.40$ & $2.06 \pm 1.41$ \\
\hline
\end{tabular}

Values are mean $\pm S D$ or ${ }^{a}$ percentage of patients

Table 2. Scoring approach for the neuropathic symptoms included in the total symptom score (pain, burning, paraesthesiae, numbness)

\begin{tabular}{lllll}
\hline Symptom frequency & \multicolumn{4}{l}{ Symptom intensity } \\
\cline { 2 - 5 } & Absent & Slight & Moderate & Severe \\
\hline Occasional & 0 & 1.00 & 2.00 & 3.00 \\
Frequent & 0 & 1.33 & 2.33 & 3.33 \\
(Almost) continuous & 0 & 1.66 & 2.66 & 3.66 \\
\hline
\end{tabular}

\section{Efficacy assessments}

Primary outcome measure. At baseline (day 1) and each subsequent visit (days 2-5, 8-12, and 15-19) prior to infusion, neuropathic symptoms (pain, burning, paraesthesiae and numbness) were scored for severity by the physician or a trained nurse. We have chosen an approach similar to a graphic rating scale [29] that uses descriptive terms to assist the patient in deciding the position of his/her score. Our approach takes into account both the intensity and frequency of each of the four symptoms in equidistant steps (Table 2). A total symptom score (TSS) ranging from 0 (no symptoms) to a maximum of 14.64 points (all symptoms are severe and [almost] continuously present) was used as a primary outcome measure. An improvement in the TSS of at least $30 \%$ (or $\geq 2$ points in patients with $\leq 4$ points at entry) from baseline to day 19 was defined as a clinically meaningful response to treatment.

Secondary outcome measures. The Neuropathy Symptom Score (NSS) was completed according to Young et al. [30] at baseline and each visit, and the Neuropathy Disability Score (NDS) [30] was assessed at baseline and on day 19 by the physician who also completed a global judgement of efficacy and tolerability of the drug (very good/good, satisfactory, inadequate, not ratable) on that day. Instruments used in the assessment of the NDS included a $128-\mathrm{Hz}$ tuning fork for measure- ment of the vibration perception threshold (VPT) on the foot (great toe, sole, dorsum, medial malleolus, mid and head of tibia) and a Thermit device (Axon, Düsseldorf, Germany) for examination of thermal sensation (identical sites, sole excepted). Abnormal VPT was defined as values less than $4 / 8$ and abnormal thermal sensation as a lack of temperature discrimination between the two plates of the device.

The Hamburg Pain Adjective List (HPAL), a multidimensional specific pain questionnaire devised by Hoppe [31], was filled in by the patient at baseline and on day 19. The HPAL includes 37 adjectives to describe pain "as it is usually". These adjectives have to be rated in seven steps ranging from "absolutely incorrect" ( 0 points) to "absolutely correct" ( 6 points). The items are summarized to four primary scales and three additional scales, respectively. Primary scales describe: 1) pain suffering by 12 items; 2) fear of pain by 9 items; 3) pain acuity by 9 items; 4) pain rhythm by 7 items. Pain suffering and fear of pain represent the affective component (21 items), while pain acuity and pain rhythm represent the sensory component (16 items). Affective and sensory components constitute additional scales. The third additional scale is the total scale ( 37 items) which provides a measure of the general pain intensity. In the analysis of the HPAL the resulting points were added separately for each scale. Increasing values indicate an increasing intensity of the individual pain component. The time needed by the patient to answer all items of the HPAL was 5$10 \mathrm{~min}$.

Laboratory methods. Glycated haemoglobin $\left(\mathrm{HbA}_{1 \mathrm{a}-\mathrm{c}}\right)$ was determined at baseline and on day 19 with the HPLC technique using a Diamat analysing system (Bio-Rad, Munich, Germany). The normal range being $<7.7 \%$ of total haemoglobin. Capillary blood glucose was taken at baseline and at each visit (days 2-5, 8-12, and 15-19) prior to the infusion and thereafter using heparinized capillary blood $(20 \mu 1)$ and was measured by the hexokinase method on an ACP 5040 autoanalyser (Eppendorf, Hamburg, Germany). 
Safety parameters were determined at baseline and on day 19 (liver enzymes, creatinine, haemoglobin, full blood count, total protein, bilirubin, uric acid, cholesterol, and triglycerides).

\section{Statistical analysis}

The continuous data listed in Table 1 are expressed by the arithmetic mean $\pm \mathrm{SD}$. The efficacy of the randomization process in balancing the groups with regard to the variables presented in Table 1 was analysed using Fisher's exact test for categorical data and ANOVA for continuous data. $P$-values less than 0.1 were considered to be a sign of inhomogeneity. The follow-up data are given as the arithmetic mean \pm SEM. Differences between groups were analysed using the $t$-test for two independent samples. The paired $t$-test was employed to test differences over time. The changes in the TSS from baseline to the follow-up visits are given on a daily basis as the arithmetic mean \pm SEM (negative values in the text signify improvement). These changes were tested between the groups on days 5,12, and 19 using the $t$-test for two independent samples. Qualitative data are given as relative frequencies which were analysed by the Fisher's exact test.

The comparison of ALA 600 with placebo regarding the response rates (improvement in the TSS by at least $30 \%$ or $\geq 2$ points in patients with $\leq 4$ points at entry) after 19 days was defined as the primary confirmatory criterion of efficacy. In addition, the time to response (i. e. onset of efficacy), as assessed by the symptom scores (burning, paraesthesiae, numbness) in the foot, was used exploratively as an alternative response criterion. Focussing on intensity only, the time to response was defined as an improvement in one of these scores by at least 1 point as compared with baseline. The time to response analysis was based on the assumption that once the response to a particular symptom occurs, the latter cannot deteriorate later. The time to response data were analysed using the Kaplan-Meier (Product Limit) estimators, and the distribution curves were compared between the groups using the log-rank (Mantel-Haenszel) test. The level of significance for invariably two-tailed testing was set uniformly at $\alpha=0.05$, and comparisons of the treatment groups were based on a per-protocol analysis in order to ascertain efficacy in patients who adhered to the protocol and the assigned treatment [32]. Only comparisons between the placebo group and each of the groups receiving $\alpha$-lipoic acid were performed.

A comparison of the time to response in one score between ALA 600 and placebo was defined as the primary criterion with which to estimate the sample size required to detect an a priori specific treatment difference. Assuming that the placebo response rate approximates $30 \%$ and that of ALA 600 yields $60 \%$ after 19 days and calculating a drop-out rate of $30 \%$, the required number of patients is estimated at $n=67$ per group, with $\alpha=0.05$ and $\beta=0.1$ for the two-tailed $\log$ rank test.

\section{Results}

Analysis of withdrawals and safety monitoring. Among the 328 patients recruited, 326 were entered into the study (ALA 1200: $n=86 /$ ALA 600: $n=77$ / ALA 100: $n=81$ /placebo: $n=82$ ), while 2 patients were not available after recruitment. Among 15 withdrawals during the study, $8(5 / 1 / 1 / 1)$ patients dropped out due to drug intolerance including mainly adverse gastrointestinal events, $2(0 / 0 / 1 / 1)$ patients due to intercurrent disease, $3(3 / 0 / 0 / 0)$ patients due to non-compliance, and $2(0 / 0 / 2 / 0)$ patients for other reasons. A further $51(13 / 13 / 11 / 14)$ patients were excluded from analysis, because they did not meet the inclusion and/or exclusion criteria. These protocol violations included age over 70 years $(n=10)$, very severe sensory deficits with reduced vibration or thermal sensation at five or six testing sites $(n=3)$, use of drugs likely to interfere with the results of the study $(n=5)$, severity less than 2 points in the TSS of one or more of the symptoms in the feet $(n=29)$, and inappropriate administration of study medication $(n=4)$. Thus, according to the protocol $260(65 / 63 /$ $66 / 66)$ patients were included in the analysis.

The rates of adverse events were 28 of $86(32.6 \%)$ in ALA 1200, 14 of 77 (18.2\%) in ALA 600, 11 of 81 $(13.6 \%)$ in ALA 100 , and 17 of $82(20.7 \%)$ in placebo. Adverse events that occurred in 5 or more patients per group included headache $(5 / 6 / 6 / 8)$ and gastrointestinal reactions which were almost restricted to ALA 1200: nausea $(13 / 2 / 1 / 1)$ and vomiting (8/0/0/ $0)(p<0.05)$ for ALA 1200 vs the remaining groups).

Glycaemic control. The mean $\mathrm{HbA}_{1}$ levels decreased from baseline to day 19 from $8.8 \pm 0.3$ to $8.6 \pm 0.3 \%$ in ALA 1200, from $9.0 \pm 0.4$ to $8.6 \pm 0.3 \%$ in ALA 600 , from $9.0 \pm 0.3$ to $8.6 \pm 0.3 \%$ in ALA 100 , and from $9.4 \pm 0.4$ to $9.0 \pm 0.3 \%$ in placebo. No significant differences were noted for the mean changes in $\mathrm{HbA}_{1}$ between the four groups studied. The blood glucose levels measured prior to the infusion on days $2-5,8-12$, and $15-18$ were significantly lower than at baseline on 4 days in ALA 1200, 1 day in ALA 600, 1 day in ALA 100, and on 3 days in placebo. The mean pre-infusion blood glucose levels on days 2-18 were $10.3 \pm 0.4 \mathrm{mmol} / \mathrm{1}$ in ALA 1200, $10.8 \pm 0.4 \mathrm{mmol} / 1$ in ALA $600,11.1 \pm 0.5 \mathrm{mmol} / 1$ in ALA 100 , and $10.4 \pm 0.3 \mathrm{mmol} / \mathrm{l}$ in placebo, without significant differences between the groups.

Symptom scores. The mean changes in the TSS as compared with baseline on a daily basis in the four groups studied are shown in Figure 1. There was a significant decrease from baseline which was first observed on days 2 or 3 in each of the four groups studied (all $p<0.05$ ). The decrease in TSS over baseline was significantly more pronounced after 5 days in ALA 1200, ALA 600, and ALA 100 than in placebo (all $p<0.01$ ) and after 12 and 19 days in ALA 1200 and ALA 600 than in placebo (all $p<0.01$ ).

The mean changes in the individual scores for burning, paraesthesiae, numbness, and pain from baseline to day 19 are illustrated in Figure 2. The reduction after 19 days was significantly higher for burning, paraesthesiae, and numbness in ALA 1200 and ALA 600 than in placebo (all $p<0.05$ ), and for pain 

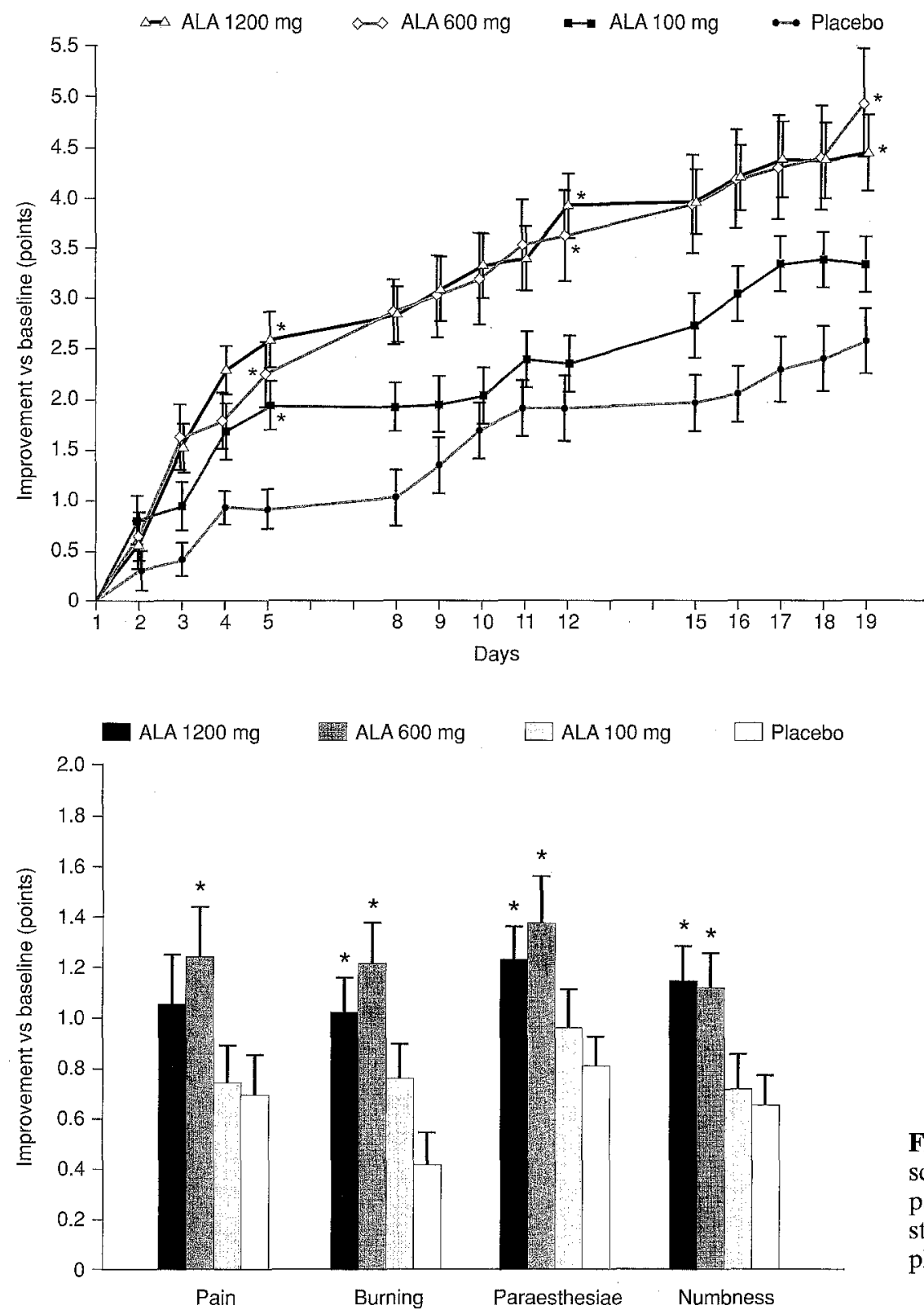

Fig. 1. Changes (improvement) in the total symptom scores (TSS) from baseline on a daily basis in the four groups studied (mean \pm SEM). $* p<0.05$ vs changes in placebo for statistical testing after 5,12 , and 18 days
Fig. 2. Changes (improvement) in the individual scores for burning, paraesthesiae, numbness, and pain from baseline to day 19 in the four groups studied (mean $\pm \mathrm{SEM}$ ). ${ }^{*} p<0.05$ vs changes in placebo in ALA 600 as compared with placebo $(p=0.02)$. There were no significant differences in the reduction of these symptoms between ALA 100 and placebo after 19 days. When additional statistical testing was performed on days 5 and 12, significant differences between ALA 1200 and placebo were noted on both days for all symptoms (all $p<0.05$ ), between ALA 600 and placebo on both days for pain and burning $(p<0.05)$ and on day 5 for numbness $(p=0.02)$, and between ALA 100 and placebo on day 5 for pain and burning $(p<0.05)$ (data not shown).

The response rates after 19 days, defined as an improvement in the TSS in the foot of at least $30 \%$ (or $\geq 2$ points in patients with $\leq 4$ points at entry) were 46 of $65(70.8 \%)$ in ALA 1200,52 of $63(82.5 \%)$ in ALA 600,43 of $66(65.2 \%)$ in ALA 100 , and 38 of $66(57.6 \%)$ in placebo $(p=0.002$ for ALA 600 vs placebo).
The time to response curves, defined as an improvement in one score (burning, paraesthesiae, or numbness) by at least 1 point (see statistical analysis), are presented in Figure 3. The rates of non-responders obtained by this definition were significantly lower after 19 days in ALA 1200 and ALA 600 as compared with placebo $(p<0.05)$, but no such difference was observed for ALA 100.

Multidimensional pain assessment. The mean changes from baseline to day 19 in the total scale of the Hamburg Pain Adjective List (HPAL) as a measure of the general pain intensity are shown in Figure 4 . The reduction of pain intensity in the total scale was significantly greater in ALA 1200 and ALA 600 than in placebo (both $p<0.01$ ), but not in ALA 100 as compared with placebo. Likewise, pain intensity in both the affective and sensory scales declined significantly 


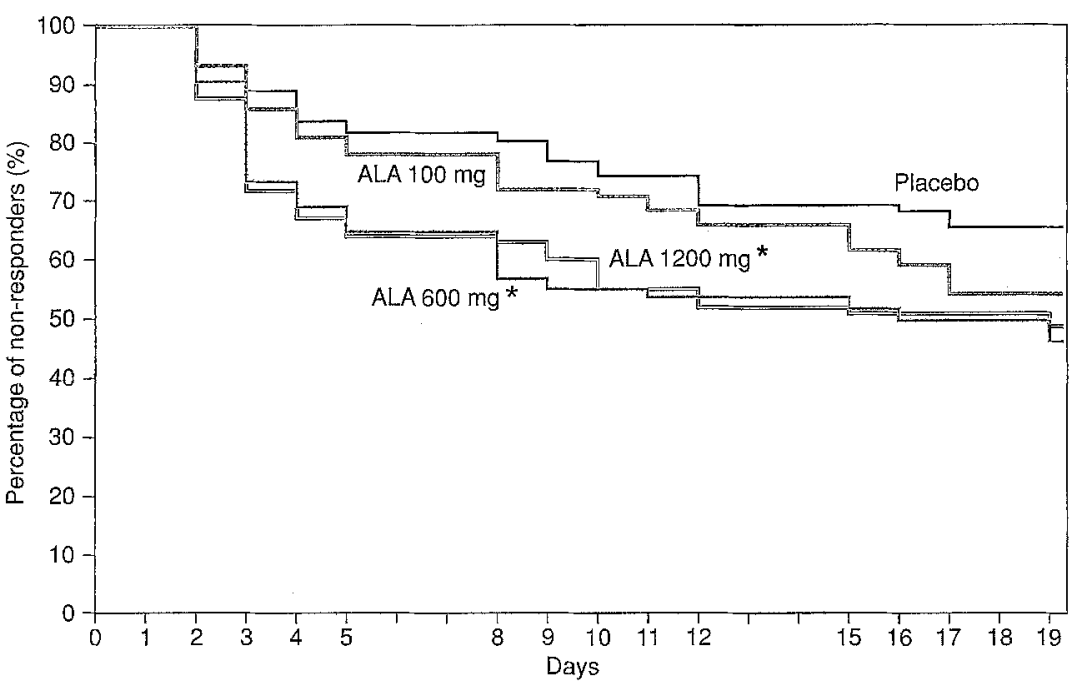

Fig. 3. Time to response curves, defined as an improvement in one score (burning, paraesthesiae, or numbness) by at least one point without deterioration during the remaining study period. The curves plotted illustrate the percentages of non-responders. ${ }^{*} p<0.05$ vs placebo

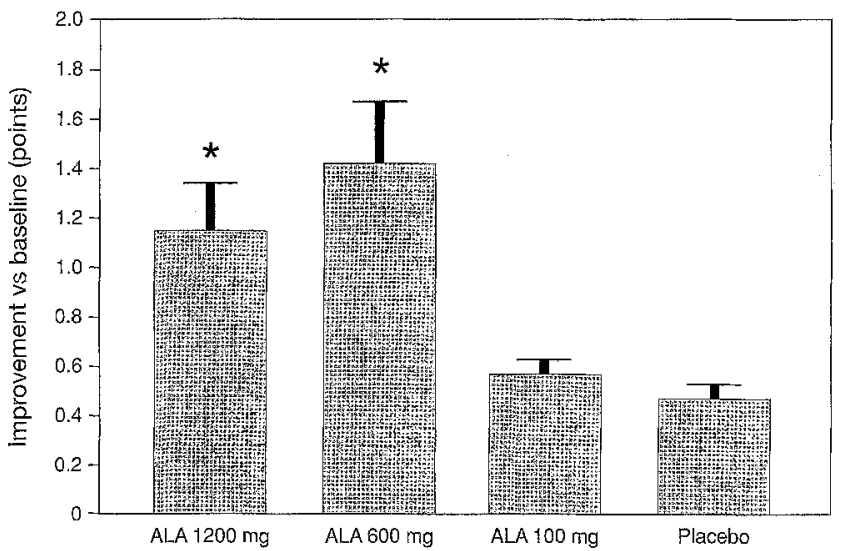

Fig.4. Changes (improvement) from baseline to day 19 of the total scale of the Hamburg Pain Adjective List (HPAL) (mean $\pm \mathrm{SEM}$ ). $* p<0.05$ vs changes in placebo

by $-1.4 \pm 0.3$ and $-0.8 \pm 0.2$ points in ALA 1200 and by $-1.6 \pm 0.3$ and $-1.1 \pm 0.2$ points in ALA 600 as compared with $-0.5 \pm 0.1$ and $-0.4 \pm 0.1$ points in placebo (all $p<0.05$ ), but the reduction by $-0.6 \pm 0.1$ and $-0.6 \pm 0.1$ points in ALA 100 was similar to placebo.

Clinical signs. After 19 days the Neuropathy Disability Score (NDS) decreased by $-1.8 \pm 0.3$ points in ALA 1200 , by $-1.5 \pm 0.3$ points in ALA 600 , by $-0.9 \pm 0.3$ in ALA 100 , and by $-1.0 \pm 0.2$ in placebo ( $p=0.03$ for ALA 1200 vs placebo).

Global rating. The percentages of patients rated as "very good/good efficacy" by the physician's global evaluation on day 19 were 40 of $65(61.5 \%)$ in ALA 1200, 48 of $63(76.2 \%)$ in ALA 600,35 of 66 $(53.0 \%)$ in ALA 100 , and 30 of $66(45.5 \%)$ in placebo ( $p=0.001$ for ALA 600 vs placebo). Overall drug tolerance rated as "very good/good" was 71 of 86 $(82.6 \%)$ in ALA 1200, 73 of $77(94.8 \%)$ in ALA 600,75 of $81(92.6 \%)$ in ALA 100 and 80 of 82 $(97.6 \%)$ in placebo.

\section{Discussion}

The findings of the present study demonstrate that parenteral treatment with the anti-oxidant $\alpha$-lipoic acid over 3 weeks using a dose of $600 \mathrm{mg}$ per day in NIDDM patients is associated with a significant reduction of various symptoms of peripheral neuropathy including pain, paraesthesiae, and numbness as compared with placebo. Furthermore, we provide evidence that a dose of $100 \mathrm{mg}$ per day does not exert an effect superior to that seen with placebo. Finally, an increase in the dosage to $1200 \mathrm{mg}$ per day is associated with an enhanced rate of adverse events rather than with maximized efficacy, since the latter was comparable to that of $600 \mathrm{mg}$ per day.

Previous double-blind controlled studies have not provided convincing data regarding the efficacy of $\alpha$-lipoic acid in diabetic peripheral neuropathy. The only controlled study hitherto published in which $\alpha$ lipoic acid was administered intravenously was conducted by Schulz et al. [33] who treated 31 insulin-dependent diabetic (IDDM) patients using $200 \mathrm{mg}$ per day i. v. over 15 days. In that study no significant effect on neuropathic symptoms was noted, but the distal motor latencies in the median, peroneal, and tibial nerves improved significantly as compared with placebo treatment. In another controlled study over 12 weeks, Jörg et al. [34] treated 35 IDDM patients with clinical or electrophysiological signs of neuropathy with $\alpha$-lipoic acid ( $600 \mathrm{mg} /$ day orally) or a vitamin B combination ( $600 \mathrm{mg} /$ day orally), respectively. No significant therapeutic effects were demonstrated for any of the parameters measured including sensory nerve conduction velocity (NCV), nerve action potentials to single and double stimuli, relative refractory period, and nerve ischaemia resistance. However, in that study neuropathic symptoms were not assessed. In a 3-week double-blind study, Sachse and Willms [35] used a relatively low oral dose of 
$300 \mathrm{mg}$ per day in 10 diabetic patients. They could not detect any differences between drug and placebo treatment for subjective symptoms, NCV or vibration perception threshold (VPT).

However, these studies had several essential drawbacks which make it impossible to ascertain whether $\alpha$-lipoic acid is an effective compound for treatment of diabetic polyneuropathy. First, in line with $95 \%$ of the previously published randomized controlled clinical trials [36], did these studies not estimate the sample size required to detect an a priori specified treatment difference. In the present trial we reasoned from previous studies a placebo response of about $30 \%$ and a drug response of about $60 \%$. The a priori estimate of the required sample size yielded $n=67$ patients per group, with $\alpha=0.05$ and $\beta=0.1$ for the two-tailed log-rank test. Thus, it becomes evident that the groups treated in the aforementioned studies [33-35] were too small to detect differences in neuropathic symptoms. Second, in view of our results, the intravenous dose of $\alpha$-lipoic acid chosen by Schulz et al. [33] was in fact too low to induce an effect. Third, the severity of the neuropathic symptoms was not scored and no detailed assessment of pain intensity was performed $[33,35]$. For these reasons, the chiefly negative results of the previous controlled studies could be anticipated.

The results of this controlled trial confirm those of our previous single-blind pilot study [28] in that they provide evidence of an improvement in the major neuropathic symptoms after 3 weeks of intravenous treatment with $600 \mathrm{mg} \alpha$-lipoic acid. This effect was noted not only for pain and paraesthesiae but also for burning and numbness, and when these symptoms were added to a total score, the difference conpared to placebo was observed after only 5 days. During the following 2 weeks a continuously sustained improvement in this score was seen. Detailed analysis of pain using a multidimensional questionnaire revealed that both the affective and sensory components of pain, representing pain experience and pain perception, could be improved. Although administration of $1200 \mathrm{mg}$ per day seemed to result in a slightly more pronounced effect than $600 \mathrm{mg}$ per day on neuropathic symptoms after 5 days, particularly paraesthesiae, the response rates after 19 days tended to be higher with $600 \mathrm{mg}$ per day. Moreover, the increased risk of gastrointestinal side effects associated with $1200 \mathrm{mg}$ per day preclude this dose being used. On the other hand, the efficacy of $100 \mathrm{mg}$ per day was not distinguishable from placebo for the majority of the parameters examined.

It is unlikely that the effects observed in this study were biased by an improvement in glycaemic control, since no significant differences between the groups treated with $\alpha$-lipoic acid and placebo were noted for the changes in $\mathrm{HbA}_{1}$ from baseline to day 19 . Moreover, no significant differences between the groups were seen regarding the blood glucose levels during the study.

It may be argued that nerve conduction studies were not used as objective measures of neuropathy in this study. However, in a short-term study of this kind a significant difference between the groups treated with $\alpha$-lipoic acid and the placebo group regarding NCV would not be likely to occur. Previous studies using drugs such as aldose reductase inhibitors have shown that NCV was either unchanged [37] or only minimally increased during several weeks of treatment [38]. The latter study [38] was subject to substantial criticism as to whether it showed a clinically meaningful degree of change or merely a physiological variation [39]. Moreover, a significant deterioration in $\mathrm{NCV}$ during placebo treatment usually does not occur even after 1 year [12]. By contrast, neuropathic symptoms have been shown to be susceptible to intervention within a few weeks. Painful symptoms, but not motor and sensory $\mathrm{NCV}$, were improved after 4 weeks of treatment with sorbinil as compared with placebo [37], and withdrawal of tolrestat resulted in a rapid worsening of pain scores [40].

It may also be argued that the exclusion of $51 \mathrm{pa-}$ tients due to failure to adhere to the protocol potentially could have introduced bias. We chose a per-protocol analysis of those patients who fulfilled the criteria for entry into the study and adhered to the assigned treatment but were aware of the fact that this approach would exclude a certain number of patients from analysis. It has recently been emphasized that an intention-to-treat (as randomized) analysis of clinical trials has no advantage over a per-protocol analysis [32]. In fact, an additional analysis of the results of this study based on the intention-to-treat revealed no appreciable differences in the outcome of the parameters studied when compared with the perprotocol analysis, indicating that the adherence to the study protocol did not introduce bias.

The exact mechanisms by which $\alpha$-lipoic acid exerts its effect on neuropathic symptoms have yet to be established, but at least two putative hypotheses should be discussed. First, $\alpha$-lipoic acid may induce a dose-dependent sprouting of neurites in cultured neuroblastoma cells [41]. Changes in membrane fluidity that are mediated by the sulphohydryl groups of the substance are thought to be responsible for this effect. In the experimentally-induced acrylamide neuropathy there is a marked reduction of the sprouting phenomenon and distal neuropathy occurs due to the depletion of substances containing sulphohydryl groups, such as glutathione, in the axon [42]. Spontaneous sprouting and integrity of the membrane at the nerve terminal can be maintained by administration of $\alpha$-lipoic acid in vivo and in vitro. Moreover, in animal experiments the compound promotes regeneration after partial denervation [42]. 
Such a protective effect of $\alpha$-lipoic acid has also been demonstrated in hexacarbon-induced neuropathy [43].

The second, and presumably most relevant, mechanism of action is based on the property of $\alpha$-lipoic acid to act as a radical scavenger. A growing body of evidence suggests that oxidative stress resulting from enhanced free-radical formation and/or defects in anti-oxidant defences is implicated in the development of various disorders [44] including neurodegenerative diseases [45]. Cellular injury induced by intracellular alterations in the metabolism of defence systems against oxidative stress may also be relevant in the pathogenesis of diabetic complications [46]. Increased free radical formation and changes in haemostatic variables related to endothelial damage have been found in NIDDM patients with microalbuminuria [47]. Furthermore, impaired cellular scavenging activity against oxidative stress has recently been demonstrated in NIDDM patients [48]. In experimental diabetic neuropathy, oxygen free radical activity in the sciatic nerve is increased $[19,20]$. Anti-oxidant treatment with butylated hydroxytoluene [24], glutathione [23], probucol [25, 26], $\alpha$-tocopherol [26], and $\alpha$-lipoic acid [27] prevents both the evolution of motor and sensory nerve conduction deficits and abnormalities in nerve blood flow in experimental diabetes. Lipophilic free radical scavengers such as $\alpha$-lipoic acid or probucol seem to be more effective than hydrophilic ones such as glutathione [21]. Conversely, treatment of non-diabetic rats with the pro-oxidant primaquine caused a reduction in nerve blood flow and NCV deficits [25]. Only recently, $\mathrm{Na}-$ gamatsu et al. [27] have demonstrated a dose-dependent normalization in digital NCV, nerve blood flow and glutathione levels following treatment with $\alpha$-lipoic acid in diabetic rats, suggesting that the improvement in neurovascular changes was induced by improving oxygen free radical scavenging activity. One mechanism of reduced nerve blood flow is the inhibitory effect of superoxide anion on nitric oxide synthase. Since nitric oxide synthase is reduced in experimental diabetic neuropathy [49], $\alpha$-lipoic acid might prevent this inhibition by reducing oxidative stress [27].

It is conceivable that the initial diabetes-related changes in the nerve are mediated by oxidative stress which, on a long-term basis, could result in progressive neuronal damage and therefore, would be of pathogenetic relevance. In the light of this, a potential basis is provided for treating diabetic neuropathy using anti-oxidants such as $\alpha$-lipoic acid. Long-term studies of oral treatment with $\alpha$-lipoic acid are needed to address the question as to whether the observed reduction in neuropathic symptoms is accompanied by long-term effects on objective neurophysiological parameters.
Acknowledgements. We are indebted to Dr. P.J.Dyck for his helpful comments on the manuscript. This study was supported by ASTA Medica AG, Frankfurt am Main, Germany.

* Participants in the ALADIN Study:

Principal investigator: D.Ziegler, Düsseldorf; study director: M.Lobisch, Frankfurt/M.; study co-ordinator: K.Schütte, Frankfurt/M.; monitoring: E.-C. Ticinelli, B.Hahnzog, Frankfurt/M.; statistics: D. Nehrdich, C. Netten, Frankfurt/M.; K. Dannehl, Düsseldorf; project leader: M.Peukert, K. Wessel, Frankfurt/M.; advisor: F. A.Gries, Düsseldorf; investigators, study sites: M. Anders, Berlin; H. Bräuning, Nauen; M.Brun, Potsdam; E.Brunner, Leipzig; S.v. Bültzingslöwen, Berlin; B.Donaubauer, Oschatz; W.Forchheim, Mühlheim/M.; K.Funke, Potsdam; S. Gerlach-Eniyew, Leipzig; T. Hampel, Schwedt; M. Hanefeld, I. Hoche, Dresden; I. Hunecke, Leipzig; C.Klinkenstein, Frankfurt/O; K.L.v. Klitzing, Frankfurt/O.; G. Kluttig, Guben; I.König, Dessau; I. Krause, Eberswalde-Finow; R. Krïger, Beeskow; U. Kunz, Potsdam, S. Mantz, Offenbach; C. Marquardt, Hohen-Neuendorf; H.P. Meißner, Berlin; M. Mende, Lauchhammer; A.Myrach-Rahn, Berlin; E. Richter, Herzberg; K.J. Ruhnau, Berlin; W. D. Ruthe, Rathenow; K. Sand, Heidenau; R. Schubert, Schkeuditz; U.Schultz, Bad Liebenwerda; M.L.Seebacher, Berlin; M. Simonsohn, Frankfurt/M.; M.Stoll, Dreieich; M. Stündel, Berlin; G. Szilleweit, Bergholz-Rehbrücke; O. Walch, Ludwigsfelde; E. Walz, Lübben; N. Wittmann, Neu-Isenburg.

\section{References}

1. The Diabetes Control and Complications Trial Research Group (1995) The effect of intensive diabetes therapy on the development and progression of neuropathy. Ann Intern Med 122: 561-568

2. Ziegler D, Mayer P, Mühlen H, Gries FA (1991) The natural history of somatosensory and autonomic nerve dysfunction in relation to glycaemic control during the first 5 years after diagnosis of type 1 (insulin-dependent) diabetes mellitus. Diabetologia 34: 822-829

3. Ziegler D, Dannehl K, Wiefels K, Gries FA (1992) Differential effects of near-normoglycaemia for 4 years on somatic nerve dysfunction and heart rate variation in type 1 diabetic patients. Diabet Med 9: 622: 629

4. Kennedy WR, Navarro X, Goetz FC, Sutherland DER, Najarian JS (1990) Effects of pancreatic transplantation on diabetic neuropathy. N Engl J Med 322: 1031-1037

5. Max MB, Lynch SA, Muir J, Shoaf SE, Smoller B, Dubner R (1992) Effects of desipramine, amitriptyline, and fluoxetine on pain in diabetic neuropathy. N Engl J Med 326: $1250-1256$

6. Sindrup SH (1994) Antidepressants in the treatment of diabetic neuropathy symptoms. Pharmacodynamic, -kinetic, and -genetic aspects. Dan Med Bull 41: 66-78

7. Henry JA, Alexander CA, Sener EK (1995) Relative mortality from overdose of antidepressants. BMJ 310: 221-224

8. Stracke H, Meyer UE, Schumacher HE, Federlin K (1992) Mexiletine in the treatment of diabetic neuropathy. Diabetes Care 15: 1550-1555

9. The Capsaicin Study Group (1991) Treatment of painful diabetic neuropathy with topical capsaicin. A multicenter, double-blind, vehicle-controlled study. Arch Intern Med 151: $2225-2229$

10. Levy DM, Abraham RR, Tomlinson DR (1991) Topical capsaicin in the treatment of painful diabetic neuropathy. N Engl J Med 324: 776 
11. Masson EA, Boulton AJM (1990) Aldose reductase inhibitors in the treatment of diabetic neuropathy. A review of the rationale and clinical evidence. Drugs 39: 190-202

12. Ziegler D, Mayer P, Rathmann W, Gries FA (1991) Oneyear treatment with the aldose reductase inhibitor, ponalrestat, in diabetic neuropathy. Diabetes Res Clin Pract 14: 63-74

13. Gregersen G (1987) Myo-inositol supplementation. In: Dyck PJ, Thomas PK, Asbury AK, Winegrad AI, Porte D (eds) Diabetic neuropathy. Saunders, Philadelphia, pp 188-189

14. Kihara M, Schmelzer JD, Poduslo JF et al. (1991) Aminoguanidine effects on nerve blood flow, vascular permeability, electrophysiology, and oxygen free radicals. Proc Natl Acad Sci USA 88: 6107-6111

15. Thomas PK (1994) Growth factors and diabetic neuropathy. Diabet Med 11: 732-739

16. Cameron NE, Cotter MA (1993) Potential therapeutic approaches to the treatment or prevention of diabetic neuropathy: evidence from experimental studies. Diabet Med 10: 593-605

17. Jamal GA (1994) The use of gamma linolenic acid in the prevention and treatment of diabetic neuropathy. Diabet Med 11: 145-149

18. Quatraro A, Roca P, Donzella C, Acampora R, Marfella R, Giugliano D (1995) Acetyl-L-carnitine for symptomatic diabetic neuropathy. Diabetologia 38: 123 (Letter)

19. Low PA, Nickander KK (1991) Oxygen free radical effects in sciatic nerve in experimental diabetes. Diabetes 40 : 873-877

20. Nickander KK, Schmelzer JD, Rohwer DA, Low PA (1994) Effect of $\alpha$-tocopherol deficiency on indices of oxidative stress in normal and diabetic peripheral nerve. J Neurol Sci 126: 6-14

21. Cameron NE, Cotter MA (1994) The relationship of vascular changes to metabolic factors in diabetes mellitus and their role in the development of peripheral nerve complications. Diab Metab Rev 10: 189-224

22. Suzuki YJ, Tsuchiya M, Packer L (1991) Thioctic acid and dihydrolipoic acid are novel antioxidants which interact with reactive oxygen species. Free Rad Res Comms 15: 255-263

23. Bravenboer B, Kappelle AC, Hamers, FPT, van Buren T, Erkelens DW, Gispen W (1992) Potential use of glutathione for the prevention and treatment of diabetic neuropathy in the streptozotocin-induced diabetic rat. Diabetologia 35: 813-817

24. Cameron NE, Cotter MA, Maxfield EK (1993) Anti-oxidant treatment prevents the development of peripheral nerve dysfunction in streptozotocin-diabetic rats. Diabetologia 36: 299-304

25. Cameron NE, Cotter MA, Archibald V, Dines KC, Maxfield EK (1994) Anti-oxidant and pro-oxidant effects on nerve conduction velocity, endoneurial blood flow and oxygen tension in non-diabetic and streptozotocin-diabetic rats. Diabetologia 37: 449-459

26. Karasu C, Dewhurst M, Stevens EJ, Tomlinson DR (1995) Effects of anti-oxidant treatment on sciatic nerve dysfunction in streptozotocin-diabetic rats; comparison with essential fatty acids. Diabetologia 38: 129-134

27. Nagamatsu M, Nickander KK, Schmelzer JD et al. (1995) Lipoic acid improves nerve blood flow, reduces oxidative stress and improves distal nerve conduction in experimental diabetic neuropathy. Diabetes Care 18: 1160-1167

28. Ziegler D, Mayer P, Mühlen H, Gries FA (1993) Effekte einer Therapie mit $\alpha$-Liponsäure gegenüber Vitamin $B_{1}$ bei der diabetischen Neuropathie. Diab Stoffw 2: 443-448
29. Scott J, Huskisson EC (1976) Graphic representation of pain. Pain 2: 175-184

30. Young MJ, Boulton AJM, Macleod AF, Williams DRR, Sonksen PH (1993) A multicentre study of the prevalence of diabetic peripheral neuropathy in the United Kingdom hospital clinic population. Diabetologia 36: 150-154

31. Hoppe F (1991) Hamburger Schmerz Adjektiv Liste (HSAL). Manual. Beltz-Verlag, Weinheim

32. Sheiner LB, Rubin DB (1995) Intention-to-treat analysis and the goals of clinical trials. Clin Pharmacol Ther 57: 6-15

33. Schulz B, Reichel G, Hüttl I, Zander E, Runge U (1986) Zur Wirksamkeit der Thioctsäuretherapie bei Typ-I-Diabetikern. Wiss Z Ernst-Moritz-Arndt-Universität Greifswald, Medizinische Reihe 35: 48-50

34. Jörg J, Metz F, Scharafinski H (1988) Zur medikamentösen Behandlung der diabetischen Polyneuropathie mit der Alpha-Liponsäure oder Vitamin B-Präparaten. Nervenarzt 59: 36-44

35. Sachse G, Willms B (1980) Efficacy of thioctic acid in the therapy of peripheral diabetic neuropathy. In: Gries FA, Freund HJ, Rabe F, Berger H (eds) Aspects of autonomic neuropathy in diabetes. Horm Metab Res [Suppl Series] 9: 105-108

36. Cavaliere D, Scorpiglione N, Belfiglio M et al. (1994) Quality assessment of randomised clinical trials on medical treatment of diabetic neuropathy. Diab Nutr Metab 7: 287-294

37. Young RJ, Ewing DJ, Clarke BF (1983) A controlled trial of sorbinil, an aldose reductase inhibitor, in chronic painful diabetic neuropathy. Diabetes 32: 938-942

38. Judzewitsch RG, Jaspan JB, Polonsky KS et al. (1983) Aldose reductase inhibition improves nerve conduction velocity in diabetic patients. N Engl J Med 308: 119-125

39. Young RR, Shahani BT (1983) Nerve conduction velocity in diabetes. N Engl J Med 308: 190-191

40. Santiago JV, Sönksen PH, Boulton AJM et al. (1993) Withdrawal of the aldose reductase inhibitor tolrestat in patients with diabetic neuropathy: effect on nerve function. $J$ Diab Comp 7: 170-178

41. Dimpfel W, Spüler M, Pierau F-K, Ulrich H (1990) Thioctic acid induces dose-dependent sprouting of neurites in cultured rat neuroblastoma cells. Dev Pharmacol Ther 14: 193-199

42. Kemplay S, Martin P, Wilson S (1988) The effects of thioctic acid on motor nerve terminals in acrylamide-poisoned rats. Neuropathol Appl Neurobiol 14: 275-288

43. Altenkirch H, Stoltenburg-Didinger G, Wagner HM, Herrmann J, Walter G (1990) Effects of lipoic adic in hexacarbon-induced neuropathy. Neurotoxicol Teratol 12: 619-622

44. Halliwell B (1994) Free radicals, antioxidants, and human disease: curiosity, cause, or consequence? Lancet 344: 721724

45. Jenner P (1994) Oxidative damage in neurodegenerative disease. Lancet 344: 796-798

46. Baynes JW (1991) Role of oxidative stress in development of complications in diabetes. Diabetes 40: 405-412

47. Collier A, Rumley A, Rumley AG et al. (1992) Free radical activity and hemostatic factors in NIDDM patients with and without microalbuminuria. Diabetes 41: 909-913

48. Yoshida K, Hirokawa J, Tagami S, Kawakami Y, Urata Y, Kondo $\mathrm{T}$ (1995) Weakened cellular scavenging activity against oxidative stress in diabetes mellitus: regulation of glutathione synthesis and efflux. Diabetologia 38: 201-210

49. Kihara M, Low PA (1995) Impaired vasoreactivity to nitric oxide in experimental diabetic neuropathy. Experimental Neurology 132: 180-185 